Trauma Surgery \& Acute Care Open

\title{
Patient-reported outcomes in trauma: a scoping study of published research
}

\author{
Graeme M Rosenberg, ${ }^{1}$ Christopher Stave, ${ }^{2}$ David A Spain, ${ }^{1}$ Thomas G Weiser ${ }^{1,3}$
}

'Department of Surgery, Section of Acute Care Surgery, Stanford University, Stanford, California, USA

2Lane Medical Library, Stanford University, Stanford, California, USA

${ }^{3}$ Department of Clinical Surgery, University of Edinburgh, Royal Infirmary of Edinburgh, Edinburgh, UK

Correspondence to Dr Graeme M Rosenberg, Department of Surgery, Stanford University, Stanford, CA 94305 , USA; gmr87@stanford.edu

Received 27 June 2018 Accepted 4 July 2018

(c) Author(s) (or their employer(s)) 2018. Re-use permitted under CC BY-NC. No commercial re-use. See rights and permissions. Published by BMJ.

To cite: Rosenberg GM, Stave C, Spain DA, et al. Trauma Surg Acute Care Open 2018:3:e000202.

\section{ABSTRACT}

More people are surviving traumatic injury, but disability and reduced quality of life are frequent. Investigators are now focusing on patient-reported outcomes (PROs) to better understand this problem. We performed a scoping study of the literature to explore trends in the study of PROs after injury. The volume of published literature on PROs after injury has consistently increased, but use of measurement tool and categorization of publications are inconsistent. Journal keyword patterns are inconsistent and likely limit the effective dissemination of important findings. In studies of hospitalized trauma patients, more than 100 unique measurement tools were used, and trauma-specific measures were used in fewer than $5 \%$ of studies. International investigators are more consistent than those in the USAin the use of validated, classic measurement tools such as the Short-Form 36 and the EuroQoL Five-Dimension tools. Uniform use of measurement tools would help improve the quality and comparability of research on PROs, and trauma-specific measures would enhance the study of long-term injury outcomes.

\section{INTRODUCTION}

The goals of a mature trauma system include injury prevention, prehospital treatment, acute hospital care, rehabilitation, and re-engagement with society. ${ }^{1-4}$ Modern US trauma systems have achieved great success in saving patients who sustain serious injuries, demonstrated by increased survivorship during the last three decades. ${ }^{5-7}$ As overall care improves, further advances in preventable deaths are likely to be small. While investigations in trauma typically focus on classic research methodologies including randomized controlled trials and database-driven clinical outcomes research to assess mortality, readmission rate, and complications,${ }^{8}$ trauma systems should now shift to more nuanced measures such as quality of life (QOL) and patient-reported outcomes (PROs). ${ }^{9}{ }^{10}$

Despite the increased survivorship, little is known about patients' long-term QOL and function. Specifically, there is a gap in understanding biopsychosocial and economic outcomes, despite growing interest in these issues. Understanding PROs and QOL after injury is increasingly relevant since trauma is common and affects all age groups, and it is expected that survivorship will continue to grow.

According to the Global Burden of Disease study, the rate of death from injury increased 11\% from 1990 to 2013 and caused an estimated 4.8 million deaths in $2013 .{ }^{11}$ It is estimated that $30 \%$ of lost years of life are due to traumatic injuries. ${ }^{12}$ Injuries affect the young, the old, rich, poor, sick, healthy, and all races, genders, and religions-one in ten people in the US population will be injured during their lifetime. ${ }^{13}$ It is often difficult to predict how long patients will be in the hospital and if they will be going home or to a long-term care or rehabilitation facilities. Predicting return-to-work, aftercare needs, and other biopsychosocial and health-related outcomes is even more difficult. Importantly, few understand the financial burden or the impact on loved ones and family members after severe injury. Because the significance and severity of injury vary widely, setting expectations and providing accurate and timely anticipatory guidance are extremely challenging.

Except for a few notable examples, there has been limited focus on studying PROs in trauma care, especially as it relates to long-term QOL and survivorship. ${ }^{9}$ To better understand the prevalence of studies involving PROs, we explored the last 30 years of trauma literature with a goal to (1) provide a broad overview of temporal trends in studying PROs in the trauma literature; (2) assess publication patterns of investigations studying PROs after operative intervention for injuries/emergencies; and (3) explore what PRO measures are being used in studies investigating patients hospitalized for injuries-excluding isolated brain/spine injuries and burns-to focus on a population largely represented by polytrauma patients. To achieve these aims, we conducted a scoping study of the available published literature.

\section{METHODS}

We performed an explorative scoping study of published literature through a query of the National Library of Medicine's PubMed database. A scoping study has been defined as a method to "map the literature on a particular topic or research area and provide an opportunity to identify key concepts; gaps in the research; and types and sources of evidence to inform practice, policymaking, and research." 1415 An often-cited framework for approaching scoping studies was developed by Arksey and O'Malley ${ }^{16}$ and includes determining the research question; identifying relevant studies; appropriate study selection; data collection and summarization; and reporting findings. These guiding principles were used in completing this scoping study. Scoping studies are ineligible for inclusion with the PROSPERO database; however, we used the principles from the Preferred Reporting Items for Systematic Reviews and Meta-Analyses checklist to guide the work. ${ }^{17}$ Article review and data extraction were 
performed by a single reviewer-data elements recorded are detailed below.

To achieve our goal of exploring trends in studies investigating PROs in trauma, we used three increasingly selective search strategies for publications available in the English language. Search language was carefully crafted using an iterative process to identify the desired content and cross-checked for inclusion of known seminal articles. Search methods and query language were conducted in collaboration with a librarian specialist with expertise in performing literature reviews. For each search, all titles were screened for obvious exclusion, followed by review of the abstract for inclusion. Articles not available in English were excluded, as were those not related to traumatic injuries. The inclusion criteria of each of the three search strategies varied based on the specific topic of interest and are detailed below. Publication characteristics, subject matter, and keywords were recorded. If data were unavailable in the abstract, the full texts were reviewed and data points recorded. Descriptive statistics were calculated using Microsoft Excel V.16.13. To estimate temporal trends in publication patterns, we calculated linear regression models of the number of publications over time for each publication type. To assess differences between publication types, we calculated a combined linear regression model including independent variables for year, publication type, and the statistical interaction. We evaluated the interaction coefficient (year $\times$ publication type) to determine whether the increase in number of publications by year differed between publication types. Statistical significance was assessed at the level of $\mathrm{p}<0.05$. Linear regression modeling was performed using Stata/MP V.14.2.

The first strategy was intentionally broad and designed to identify all types of publications discussing PROs from injured patients between 1985 and 2015 (online supplementary content 1.1; detailed search language). Publications beyond 2015 were not included to ensure complete indexing of available articles. This search was performed on May 1, 2017. Articles were included if they discussed PROs or quality of life in the title or abstract. Only articles pertinent to injuries were included. The change in volume of publications over 30 years was quantified and compared with related publication patterns in the broader categories of "injuries" and "patient-reported outcomes" during the same time period. By comparing these broader publication patterns, the goal was to ascertain if PRO research in injured patients has kept pace with broader benchmark categories and topics. The comparison search strategies were performed on June 26, 2017 (online supplementary content 1.2 and 1.3; detailed search language).

The second search strategy specifically limited the original search to articles discussing PROs after operative management for injuries and surgical emergencies. Article cataloging and keywording was dissimilar between surgical specialties; therefore, the resulting search includes articles pertaining to operative management of surgical emergencies as it was functionally difficult to limit specifically to injuries. The intention was to assess how the various surgical specialties were contributing to the study of PROs. For this search, we excluded systematic reviews, meta-analyses, letters/editorials, and case reports with a sample size less than 18 patients. This search was performed on May 1, 2017 (online supplementary content 2; detailed search language).

The third search was constructed to identify the most recent PRO literature describing studies of patients with moderate to severe injuries from 2007 to 2017 (online supplementary content 3; detailed search language). An iterative approach was
Box 1 Exclusion criteria for the third search strategy

Case reports $(n<18)$.

- Review articles, systematic reviews, meta-analysis and guidelines.

- Non-injury trauma (psychological trauma).

- Assessment of future risk of injury.

- Joint/ligamentous injuries.

- Osteoporotic/arthritis-related injuries.

- Degenerative joint disease and related injuries.

- Concussion/mild traumatic brain injury/acquired brain injury.

- Isolated dental injuries.

- Isolated ocular injuries.

- Whiplash.

- Mild occupational injuries.

Mild sports-related/athletic injuries.

used to confirm that the desired subject matter was identified and that known formative and influential work was captured by the search language. Since the science and interest of studying PROs continue to improve, we focused on the last decade of published research. Our intention was to evaluate the literature regarding moderate/severe injuries and polytrauma given the greater likelihood for long-term disability with accompanying biopsychosocial and financial impact-exclusions are found in box 1. Further, the search strategy was designed to exclude post-traumatic distress from psychological trauma, unless discussed specifically in relation to a traumatic injury. To explore which PRO measures were being used to study the population of significantly injured individuals, we restricted our final analysis to studies of injured patients requiring hospitalization while excluding minor sports-related and occupational injuries. Studies of patients with burns and isolated injuries to the brain/spine were also excluded in an attempt to focus on polytraumatic injuries. Fortunately, the existing literature regarding PROs and QOL in these excluded isolated injury populations is robust and well reviewed. These article types were categorized and excluded from the final subset of articles. In this resulting subset of research articles, the specific PRO measures used were recorded. The search was performed on June 26, 2017.

\section{RESULTS}

We identified 12903 publications between 1985 and 2015 evaluating PROs in injured patients. The studies included 2241 (17.4\%) reviews/meta-analyses, 1401 (10.9\%) clinical trials, 948 $(7.3 \%)$ randomized controlled trials, 896 (6.9\%) case reports, and $285(2.2 \%)$ letters/editorials/comments. The remaining $7132(55 \%)$ articles were original research. The annual number of publications exploring PROs for injured patients increased in a linear fashion (figure 1, gray bars) and was 27.8 times higher in 2015 than in 1985 . The magnitude in change of the publication volume is higher than that of publications in the more general category "wounds \& injuries", which grew $3.6 \times$ (figure 1, black bars). The publication volume in the broad category of "patient report outcome \& quality of life" grew 19.9-fold during the period of 30 years. During the same time interval, the overall volume of English literature published in PubMed grew fourfold. The rate of increase, however, was significantly slower for publications relating to "PROs after injury" as judged by a trendline slope of 41.8 compared with a slope of 65.6 for the broader category of "wounds \& injuries" $(\mathrm{p}<0.001)$. 


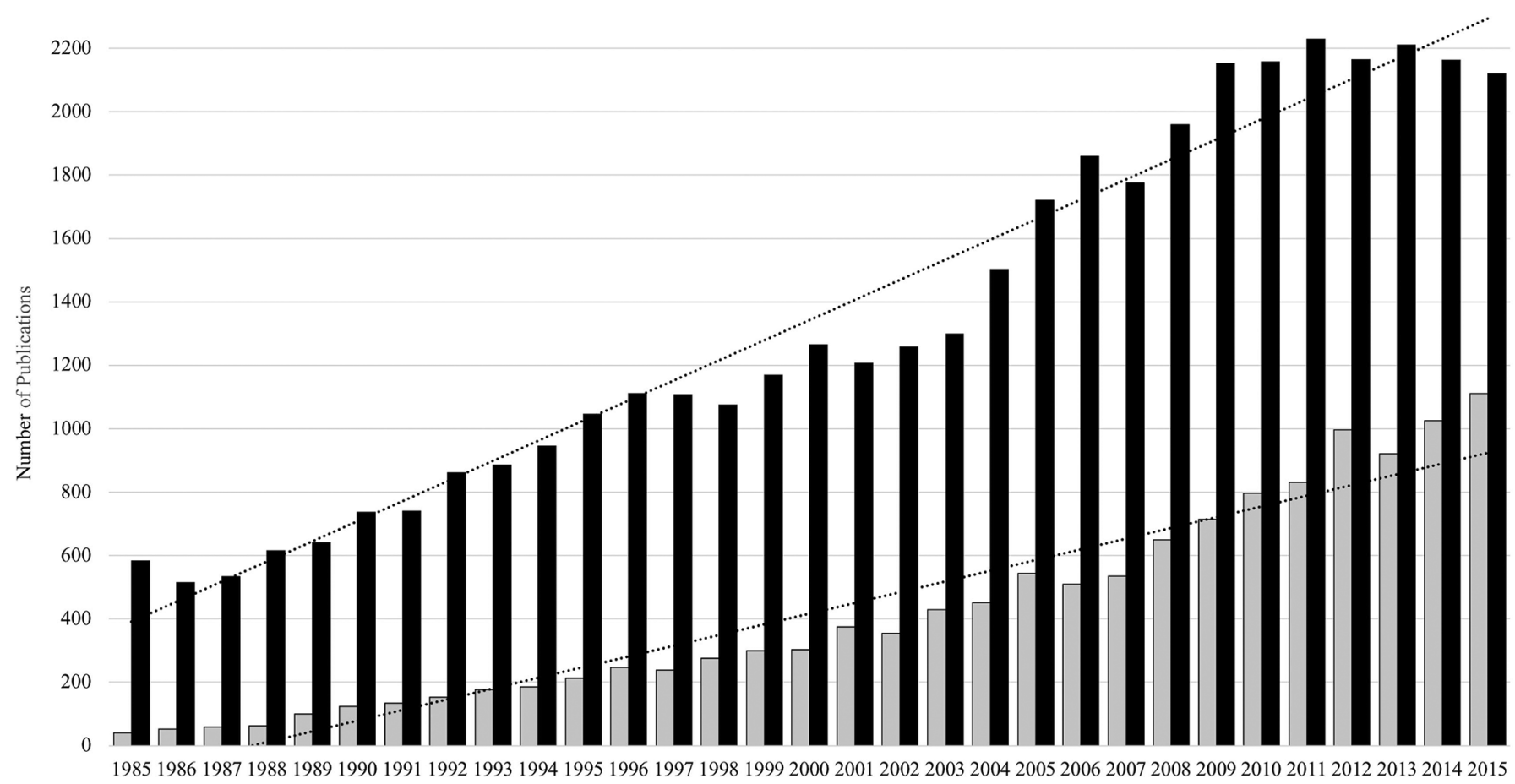

Figure 1 A comparison of annual publications of articles studying "injuries" (black bars) and "patient-reported outcomes after traumatic injury" (gray bars) between 1985 and 2015. The slopes of the trend lines fit to the graphs are 63.4 (black bars) and 33.8 (gray bars) with $\mathrm{r}^{2}=0.96$ and $\mathrm{r}=0.92$, respectively.

The second search limited the exploration of published studies to original research on PROs after operative intervention for injuries/emergencies. We identified 1134 publications, of which 475 met all the inclusion criteria (figure 2A). These articles were dominated by the orthopedic literature $(n=190,40 \%)$, followed by general surgery $(n=78,18 \%)$, plastics/hand surgery $(n=72$, $15 \%)$, and cardiothoracic surgery $(n=36,8 \%)$. The degree of heterogeneity in topic, methodology, patient population, and specialty-specific PRO instruments was high. As such, a more detailed assessment and quantitative synthesis of these articles was not undertaken as compatibility of the included studies precluded effective comparison.

The third and most focused search identified 1059 original research articles describing studies of PROs from individuals with moderate to severe injuries from 2007 to 2017. Figure 2B demonstrates the inclusion and exclusion of articles for data collection. Non-US literature predominated, representing 64\% $(n=677)$ of all articles. Before exclusion of specialty articles, four major categories were identified-traumatic brain injury which comprised $32 \%(n=336)$ of the published studies, injury to the spinal column which made up $31 \%(n=332)$, burns which accounted for $12.5 \%(n=132)$, and $25 \%(n=259)$ "trauma". Trauma” was defined as injuries to body regions other than isolated brain or spinal cord or burns (ie, pelvic injuries, extremity vascular injury, facial injury) and included polytraumatic injuries. Table 1 shows the percent breakdown of international and domestic articles for each major category and highlights that international investigators prominently contribute to the discussion of PROs after injury.

To explore the use of PRO and QOL measures, all articles in the "trauma" category were reviewed for the specific measures used and common legacy tools, as well as novel measures were recorded. The Rand Short-Form 36 Health Survey (SF-36, RAND Corporation, California, USA $)^{15} 18$ and the EuroQoL Five-Dimension questionnaire (EQ-5D, EuroQoL Group,
Rotterdam, The Netherlands) ${ }^{15} 1920$ were abstracted individually due to their prevalence of use, validity in the injury population, and frequent appearance in titles and keywords. The following additional tools were recorded given their predominance in the literature and high-quality validation: Rand Short-Form 12 Health Survey, WHO Quality of Life measure, Sickness Impact Profile, Hospital Anxiety and Depression Scale, and the Glasgow Outcomes Scale \pm Extended (GOS/GOS-E). The use of other validated tools was also recorded, but non-validated, unnamed measures or measures constructed for use during a specific study or in a specific clinic or hospital were not included. Eighty-nine percent of studies used an identifiable measure. The SF-36 was most popular, used in $36 \%$ of all studies. Table 2 shows the frequency of use of the included measures in both the international and US literature and demonstrates that international articles more consistently include either the SF-36 or EQ-5D as part of the study design.

Analysis of the keywords and phrases collected from the included articles revealed that 323 unique keywords or phrases were used to categorize the included articles. "Psychology" was the most frequently used keyword followed by "quality of life," "epidemiology," "rehabilitation," and "surveys and questionnaires," in the order of decreasing frequency. The majority of keywords were applied to only a very small fraction of articles.

\section{DISCUSSION}

Patient-reported outcomes and assessments of QOL offer a valuable lens from which to gauge treatment effects, understand the way patients experience care, and determine if individuals achieve personally acceptable outcomes. They also afford healthcare professionals and health systems the ability to improve the quality and value of the care they deliver. There has been an increased interest in and efforts toward studying PROs in all aspects of healthcare. Understanding recovery of surgical 


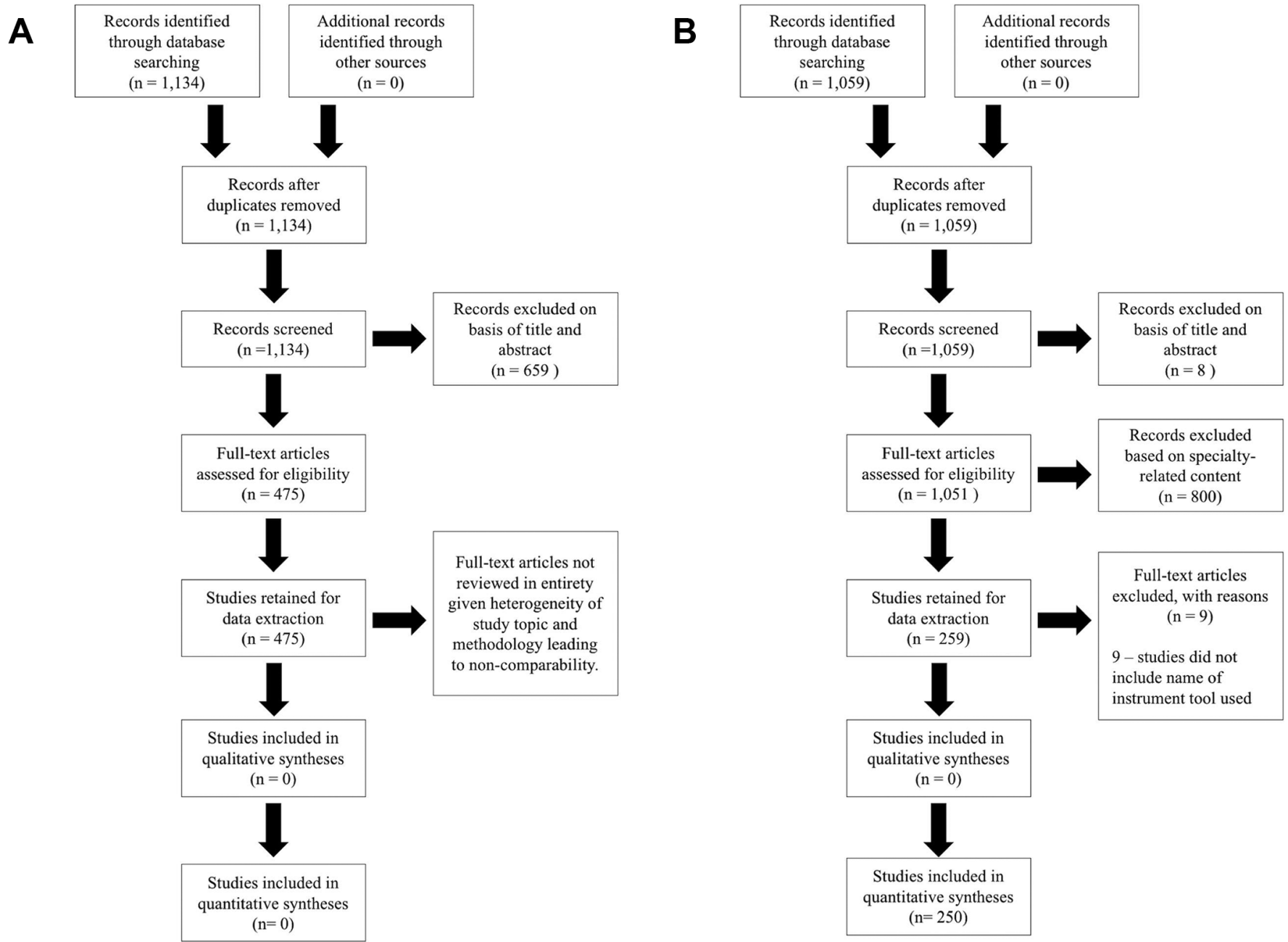

Figure 2 Preferred Reporting Items for Systematic Reviews and Meta-Analyses flow sheet of article selection process for (A) articles investigating patient-reported outcomes after operative intervention for injuries/emergencies and (B) articles investigating patient-reported outcomes after moderate to severe injuries.

patients using PROs and QOL health assessment tools is ripe for study, particularly for those suffering traumatic injury.

Through our iterative search strategy, we found that publication of articles involving PROs in injured patients has consistently increased during the last 30 years. However, it has not kept pace with studies of PROs more generally. Although the increased attention to studying PROs for injured patients is promising, we encourage continued attention to improving our understanding of PROs, QOL, and the patient experience after injury.

The study of PROs after injury is dominated by the orthopedic field; $40 \%$ of studies during the last 30 years pertain to

Table 1 Proportion of publications related to the four major categories of traumatic injuries

\begin{tabular}{llrr}
\hline & Overall & Domestic & International \\
\hline Burns & $132(32 \%)$ & $34(26 \%)$ & $98(74 \%)$ \\
\hline Traumatic brain injury & $336(32 \%)$ & $141(42 \%)$ & $195(58 \%)$ \\
\hline Spinal cord injury & $332(31 \%)$ & $123(37 \%)$ & $209(63 \%)$ \\
\hline Trauma & $259(24 \%)$ & $84(32 \%)$ & $175(68 \%)$ \\
\hline
\end{tabular}

A comparison of international articles with the domestic (USA) literature is included. "Trauma" is defined as injuries to body regions other than isolated brain or spinal cord injuries or burns and includes polytraumatic injuries. this specialty. This is likely due to a wider spectrum of activity-impacting injuries (simple fractures, ligamentous injuries, sports injuries, and so on); it is also likely due to the mechanical and functional nature of such injuries, which lend themselves to objective evaluation. It was challenging, however, to extract article pertaining to the operative management of injuries given inconsistencies in keyword categorization and title nomenclature. Surgical specialties are increasing the study of PROs and QOL to provide valuable information to the process of care. ${ }^{21}$ Multiple surgical specialties are involved in the care of traumatic injuries; therefore, consistency in how all approach the study of PROs will enhance the overall benefit of such investigations.

Table 2 Comparison of international and domestic use of classic measurement tools for studying patient-reported outcomes (PROs) in hospitalized trauma patients (excluding isolated brain/spine injuries and isolated burn injuries)

\begin{tabular}{lccc}
\hline & $\begin{array}{l}\text { Overall } \\
(\mathbf{n}=\mathbf{2 5 0})\end{array}$ & $\begin{array}{l}\text { International } \\
(\mathbf{n}=\mathbf{1 7 4}, \mathbf{7 0} \%)\end{array}$ & $\begin{array}{l}\text { Domestic } \\
(\mathbf{n}=76,30 \%)\end{array}$ \\
\hline Any PRO tool & $222(89 \%)$ & $152(87 \%)$ & $70(92 \%)$ \\
\hline Short-Form 36 & $90(36 \%)$ & $69(40 \%)$ & $21(28 \%)$ \\
\hline $\begin{array}{l}\text { EuroQoL Five-Dimension } \\
\text { questionnaire }\end{array}$ & $38(15 \%)$ & $35(20 \%)$ & $3(4 \%)$ \\
\hline
\end{tabular}


In our most selective search, the PRO trauma literature is dominated by traumatic brain and spinal cord injuries. Extensive and high-quality literature exists exploring the long-term biopsychosocial impact of brain/spinal cord injuries as individuals recover and begin re-engagement with the society. Burn injury studies are also strongly represented, whereas polytrauma is less thoroughly characterized. All specialties involved with caring for trauma patients should assess PROs as these will promote a better understanding of how patients perceive their recovery and increase clinicians' understanding of how to both obtain favorable outcomes and appropriately counsel injured patients.

Our evaluation highlights the common use of the SF-36 and EQ-5D by the international community. These two classic instruments are robustly validated for use in most patient populations. Articles published by investigators in the USA use common, well-validated instruments less consistently. Overall, more than 100 unique measurement tools were used, decreasing crossstudy comparison. Effort should be taken to use common, validated measurement tools in future investigations. Use of similar measurement tools increases the ability to compare studies and perform meta-analyses. We recommend the routine use of modern, validated psychometric measures to improve the rigor of studying relevant outcomes. One contemporary toolkit is the National Institutes of Health (NIH)-PROMIS (Patient-Reported Outcomes Measurement Information System), which provides a well-researched and carefully developed catalog of measures capable of collecting a variety of PROs in the domains of physical, mental, and social health. This toolkit is being promoted in the surgical literature. ${ }^{15202223}$ In developing the toolkit, the goal of the NIH-PROMIS was to create a set of flexible, precise, and publicly available PRO measurement tools to promote clinical research of PROs and health-related QOL. The development of the PROMIS tools leveraged modern improvements in psychometric methodology called item-response theory. ${ }^{23} 24$ The development and validation of the PROMIS tools took place on a large-scale measuring both sick and healthy patients to provide increased accuracy and responsiveness of instruments. Additionally, PROMIS tools have been rigorously compared and linked to legacy tools including the SF-36, allowing comparisons across studies and benchmarking against the normal, non-injured population using the PROsetta Stone project. ${ }^{25} 26$

Although instruments such as the SF-36, EQ-5D, and instrument catalogs like the PROMIS measures offer excellent performance in measuring general health assessments and health-related QOL, investigators studying injured patients should consider including validated, trauma-specific measures. Disease-specific measures are advantageous because they are designed to stratify patients more accurately and are better able to demonstrate changes over time and response to interventions. Validated trauma-specific tools include the Trauma Quality of Life Measure (USA) and the Trauma Outcome Profile (Germany). ${ }^{26}{ }^{27}$ Trauma-specific instruments were used in only $4 \%$ of injury studies, suggesting that they are vastly underutilized. Using trauma-specific measures will enhance the study of long-term outcomes after injury. Paired with a commonly used generic instrument, outcomes would be both trauma-specific and interpretable against an average, normative healthy comparison population.

Our assessment of the literature revealed wide variability in the way studies are classified. This presents a particular challenge to performing systematic reviews as it limits the likelihood that all pertinent studies will be identified. Inconsistencies in cataloging limit the likelihood that pertinent studies are identified, complicate dissemination of new finding, and decrease article viewing and citation. We are encouraged by the recent adoption of new "medical subject heading" (MeSH) terms "patient outcome assessment" in 2014 and "patient reported outcome measures" introduced in $2017 . .^{28} \mathrm{MeSH}$ terms are curated by the National Library of Medicine as a thesaurus of vocabulary to catalog studies together. ${ }^{29}$ Utilizing MeSH terminology greatly enhances the ease of searching the available literature. In addition to consistent use of MeSH terms to facilitate the cataloging of PRO research, we recommend that researchers begin to use common keyword vocabulary. Consistent use of a common keyword vocabulary will help to codify articles published pertaining to PROs and QOL. We suggest the use of the terms "patient-reported outcomes" and "quality of life" or "health related quality of life" be routinely used as this will enhance the categorization of PRO articles and facilitate searchability and dissemination. To assist in identifying the population of patients studied, such as trauma patient population, we recommend consistently including pertinent identifiers such as "trauma" or "emergency general surgery".

Enhancing categorization of PRO studies will improve the ability of the research community to disseminate their findings to other researchers and clinicians, and ideally quicken the pace at which findings affect patients and improve QOL. Research finding must also be disseminated to other important stakeholders, including patients, caregivers, and communities, as literature directed toward the scientific community is often inaccessible to the public or difficult to interpret. Involving patient stakeholders and sharing findings with patients is a tenant of PRO research, ${ }^{30}$ and we encourage innovative strategies to do this, such as using partnerships between the media departments of hospital systems, alignment with the press, and creative use of social media. Not only do patients deserve to be aware of research findings, but improved dissemination to patients will likely improve the pace of innovation and adoption and hopefully hasten improvements in PROs and QOL.

Clinicians, patients, and communities will benefit from an improved understanding of the effects injury has on the lives of injury survivors. We anticipate that through rigorous efforts and persistent study, we will identify factors supporting better recovery and improved QOL after injury. Undoubtedly, policy improvements and systems-level innovations will increase the chances of favorable long-term outcomes for injury survivors. The ramifications of the societal cost of injury may one day be lessened if survivors are better equipped to reintegrate as fully as possible into their communities.

Acknowledgements The authors would like to thank the research collaborators at the Stanford-Surgery Policy Improvement Research and Education (S-SPIRE) Center, specifically Dr Amber Trickey for her statistical expertise.

Contributors GMR was responsible for conception and design; data collection/ abstraction; data analysis; interpretation of analysis; drafting the article; and critical revision of important intellectual content. CS was responsible for literature search, including creation of detailed search language and confirmation of included publication type; drafting of pertinent methodological content; and critical revision of important intellectual content. DAS was responsible for conception and design; interpretation of analysis; and critical revision of important intellectual content. TGW was responsible for conception and design; methodological oversight; interpretation of analysis; and critical revision of important intellectual content. All authors have made substantial contributions to the final approval of the submitted version.

Funding The authors have not declared a specific grant for this research from any funding agency in the public, commercial or not-for-profit sectors.

Competing interests None declared.

Patient consent Not required.

Provenance and peer review Not commissioned; externally peer reviewed.

Data sharing statement Detailed search language is available in the online supplementary online content to access all pertinent articles. 
Open access This is an Open Access article distributed in accordance with the Creative Commons Attribution Non Commercial (CC BY-NC 4.0) license, which permits others to distribute, remix, adapt, build upon this work non-commercially, and license their derivative works on different terms, provided the original work is properly cited and the use is non-commercial. See: http://creativecommons.org/ licenses/by-nc/4.0

\section{REFERENCES}

1. The Committee on Trauma. Part 5: The Time Is Now: Creating and Sustaining a Unified, Learning Trauma System. 2018. https://www.facs.org/quality-programs/ trauma/trauma-series/part-v (accessed 21 Mar 2018).

2. National Academies of Sciences, Engineering, and Medicine. A National Trauma Care System: Integrating Military and Civilian Trauma Systems to Achieve Zero Preventable Deaths After Injury. Washington, DC: The National Academies Press, 2016.

3. Berwick DM, Downey AS, Cornett EA. A National Trauma Care System to Achieve Zero Preventable Deaths After Injury: Recommendations From a National Academies of Sciences, Engineering, and Medicine Report. JAMA 2016;316:927-8.

4. Jenkins DH, Winchell RJ, Coimbra R, Rotondo MF, Weireter LJ, Bulger EM, Kozar RA, Nathens AB, Reilly PM, Henry SM, et al. Position statement of the American College of Surgeons Committee on Trauma on the National Academies of Sciences, Engineering and Medicine Report, A National Trauma Care System: Integrating Military and Civilian Trauma Systems to Achieve Zero Preventable Deaths After Injury. J Trauma Acute Care Surg 2016;81:819-23.

5. Nathens AB, Brunet FP, Maier RV. Development of trauma systems and effect on outcomes after injury. Lancet 2004;363:1794-801.

6. Haas B, Jurkovich GJ, Wang J, Rivara FP, Mackenzie EJ, Nathens AB. Survival advantage in trauma centers: expeditious intervention or experience? J Am Coll Surg 2009;208:28-36.

7. Mullins RJ, Mann NC. Population-based research assessing the effectiveness of trauma systems. J Trauma 1999;47(3 Suppl):S59-S66.

8. O'Boyle CA. Assessment of quality of life in surgery. Br J Surg 1992;79:395-8.

9. Rios-Diaz AJ, Lam J, Zogg CK. The Need for Postdischarge, Patient-Centered Data in Trauma. JAMA Surg 2016;151:1101-2.

10. Haider AH, Dankwa-Mullan I, Maragh-Bass AC, Torain M, Zogg CK, Lilley EJ, Kodadek LM, Changoor NR, Najjar P, Rose JA, et al. Setting a National Agenda for Surgical Disparities Research: Recommendations From the National Institutes of Health and American College of Surgeons Summit. JAMA Surg 2016;151:554-63.

11. Haagsma JA, Graetz N, Bolliger I, Naghavi M, Higashi H, Mullany EC, Abera SF, Abraham JP, Adofo K, Alsharif $U$, et al. The global burden of injury: incidence, mortality, disability-adjusted life years and time trends from the Global Burden of Disease study 2013. Inj Prev 2016;22:3-18.
12. National Trauma Institute. 2017. http://nationaltraumainstitute.net/home/trauma_ statistics.html (accessed 14 Nov 2017).

13. Davis KA, Fabian TC, Cioffi WG. The Toll of Death and Disability From Traumatic Injury in the United States-The "Neglected Disease" of Modern Society, Still Neglected After 50 Years. JAMA Surg 2017;152:221-2.

14. Daudt HM, van Mossel C, Scott SJ. Enhancing the scoping study methodology: a large, inter-professional team's experience with Arksey and O'Malley's framework. BMC Med Res Methodol 2013;13:48.

15. Jones RS, Stukenborg GJ. Patient-Reported Outcomes Measurement Information System (PROMIS) Use in Surgical Care: A Scoping Study. J Am Coll Surg 2017;224:245-54.

16. Arksey H, O'Malley L. Scoping studies: towards a methodological framework. Int J Soc Res Methodol 2005;8:19-32.

17. Moher D, Shamseer L, Clarke M, Ghersi D, Liberati A, Petticrew M, Shekelle P, Stewart LA, PRISMA-P Group. Preferred reporting items for systematic review and metaanalysis protocols (PRISMA-P) 2015 statement. Syst Rev 2015;4:1.

18. 36-Item Short Form Survey (SF-36). 2017. https://www.rand.org/health/surveys_tools/ mos/36-item-short-form.html (accessed 18 Jul 2017).

19. The EuroQol Group. EQ-5D. https://euroqol.org/ (accessed 18 Jul 2017).

20. Smith AB, Schwarze ML. Translating Patient-Reported Outcomes From Surgical Research to Clinical Care. JAMA Surg 2017;152:811.

21. Church J. Quality of life and patient-reported outcomes. Br J Surg 2018;105:157-8.

22. Patel AA. Patient-Reported Outcome Measures: The Promise of PROMIS. J Am Acad Orthop Surg 2016;24:743.

23. HealthMeasures. PROMIS. http://www.healthmeasures.net/explore-measurementsystems/promis (accessed 18 Jul 2017).

24. Amtmann D, Cook KF, Johnson KL, Cella D. The PROMIS initiative: involvement of rehabilitation stakeholders in development and examples of applications in rehabilitation research. Arch Phys Med Rehabil 2011;92(10 Suppl):S12-S19.

25. PROsetta Stone. 2017. http://www.prosettastone.org/Pages/default.aspx (accessed 20 Jul 2017).

26. Wanner JP, deRoon-Cassini T, Kodadek L, Brasel K. Development of a trauma-specific quality-of-life measurement. J Trauma Acute Care Surg 2015;79:275-81.

27. Attenberger C, Amsler F, Gross T. Clinical evaluation of the Trauma Outcome Profile (TOP) in the longer-term follow-up of polytrauma patients. Injury 2012;43:1566-74.

28. PCORI. 2017. https://www.pcori.org/ (accessed 10 Jul 2017).

29. National Library of Medicine. MeSH Browser. 2017. https://meshb.nlm.nih.gov/ treeView (accessed 20 Jul 2017).

30. National Library of Medicine. MeSH Database. 2018. https://www.nlm.nih.gov/bsd/ disted/pubmedtutorial/020_490.html (accessed 11 Jun 2018). 